\title{
miR-29b-3p promotes progression of MDA-MB-231 triple-negative breast cancer cells through downregulating TRAF3
}

\author{
Bao Zhang, Dattatrya Shetti, Conghui Fan and Kun Wei* (i)
}

\begin{abstract}
Background: Breast cancer is the second common malignant cancer among females worldwide. Accumulating studies have indicated that deregulation of miRNA expression in breast cancer will contribute to tumorigenesis and form different cancer subtypes. However, the reported studies on miR-29b-3p-regulated breast cancer are limited so far. Herein, we investigated the role and mechanism of miR-29b-3p in the triple negative breast cancer cell line MDA-MB-231.
\end{abstract}

Methods: The relative miR-29b-3p expression in different breast cancer cell lines were determined by qRT-PCR. CCK8 and colony formation assay were used to determine the influence of miR-29b-3p on cell proliferation. Migration assay and invasion assay were performed for cell migration and invasion respectively. To study the cell integrity immunofluorescence was performed. TUNEL assay, flow cytometry assay, hoechst staining and western blot were conducted to determine the influence of miR-29b-3p inhibitor on cell apoptosis. TRAF3 was found to be the target gene of miR-29b-3p using bioinformatics predictions. Dual-luciferase assay was performed to determine the relative luciferase activity in NC, miR-29b-3p mimic, miR-29b-3p inhibitor with TRAF3 3'-UTR wt or TRAF3 3'-UTR mt reporter plasmids. The proteins expression of NF-KB signaling pathway in MDA-MB-231 after transfection with NC, miR-29b-3p mimic, miR-29b-3p inhibitor were determined by western blot.

Results: The miR-29b-3p expression was significantly increased in MDA-MB-231 compare with MCF-10A. miR29b-3p inhibitor reduced the cell viability of MDA-MB-231 and inhibited cell migration and invasion. Cell cytoskeleton integrity destroyed after miR-29b-3p inhibitor treatment. Furthermore, we identified the mechanism and found miR29b-3p targets the TRAF3 and activates NF-KB signaling pathway.

Conclusions: From the above studies, our results indicated that miR-29b-3p acts as a promoter for the development of MDA-MB-231.

Keywords: miR-29b-3p, Triple negative breast cancer, Cytoskeleton, TRAF3, NF-KB

\section{Background}

Breast cancer is considered to be one of the most common malignant cancer among females worldwide. According to the American Cancer Society, breast cancer accounts for $30 \%$ of all new cancer cases and is a major cause of cancer-related mortality in females [1-3]. In China, around $12.2 \%$ breast cancer cases have

*Correspondence: weikun@scut.edu.cn

School of Biology and Biological Engineering, South China University

of Technology, Guangzhou 510640, People's Republic of China been diagnosed and about $9.6 \%$ death cases have been reported from breast cancer worldwide [4]. In addition, the 5 -year survival rate $(68 \%)[5,6]$ is much lower than that in the United States (90\%) [2]. Attributed to improvements in early diagnosis and follow-up on effective treatments the mortality rate of breast cancer has decreased since 1987. However, the incidence of breast cancer has been increasing year by year in developing countries [2]. In China, incidence rate is growing at more than twice the rate of global growth, especially in urban areas [4]. Triple negative breast cancer (TNBC) is 
a subtype of breast cancer which lack of estrogen receptor, progesterone receptor and low expression of human epidermal growth factor receptor 2 (HER 2) [7-9]. Due to these, the prognosis was worse than other subtypes of breast cancer. Therefore, finding new biomarkers and developing effective therapeutic strategies for TNBC is instantly needed.

microRNA is a kind of small, 19-22 nucleotides in length, non-coding RNA which play a crucial role in the regulation of gene expression by binding to the $3^{\prime}$-untranslated region (UTR) of target mRNA [10-12]. Recent studies have demonstrated that miRNAs involved in many biological processes including cell proliferation, differentiation, apoptosis, development of organisms and pathogenesis of cancer [13-16]. It has been reported that miR-15a/miR-16-1 cluster act as a tumor suppressor in chronic lymphocytic leukemia (CCL) because of this they are often been deleted on the chromosome [17]. Zhang et al. [18] showed that miR-21 binds to PTEN and promotes tumor proliferation and invasion in gastric cancer. In breast cancer, many miRNAs levels are dysregulated, such as miR-210, miR-19b, miR-93 are downregulated and miR-10b, miR-145, miR-195 are upregulated, causing many functional disorders [19-22]. Therefore, miRNAs could be novel therapeutic molecular targets for breast cancer treatment.

miR-29b, one of miR-29 family members, has been reported to serve as a promoter among various kinds of disease. Zhang et al. [23] investigated that miR-29b is an effective positive regulator of adipogenesis which causes obesity. Maegdefessel et al. [24] reported that inhibition of miR-29b reduces murine abdominal aortic aneurysm development. Yang et al. [25] reported that miR-29b is a cell-cell adhesion regulator which promoted oral squamous cell carcinoma cell migration and downregulated CX3CL1. Liu et al. [26] found that miR29b levels were significantly elevated in AML leukemia stem cells compared with non-leukemia stem cells and the miR-29b silencing resulted in increased Sp1 in AML cells, it is a promoter in acute myeloid leukemia. Langsch et al. [27] found that miR-29b protects NSCLC cells from extrinsic apoptosis and mediates NF- $\mathrm{KB}$ signaling in KRAS-Induced Non-Small Cell Lung Cancers. Jarline et al. identified the miRNAs related to variations in DNA repair capacity levels in BC cases. miR-29b-3p has a higher expression in $\mathrm{BC}$ cases with low DNA repair capacity levels which positively associated with $\mathrm{BC}$ risk [28]. The members of miR-29b family are miR-29b-3p, miR-29b-1-5p, miR-29b-2-5p, and they have the high similarity of mature sequences. Despite it, distinct functions were identified because of the different isoforms [29-32].
In this study, we investigated the role of miR-29b-3p expression in triple negative breast cancer cell. Furthermore, potential targets of miR-29b-3p was identified by bioinformatics analysis and dual-luciferase reporter assay. Our study revealed that inhibition of miR-29b-3p drastically reduces cell proliferation, migration, invasion in TNBC. Moreover, inhibiting miR-29b-3p also induces apoptosis in TNBC through PARP cleavage. However, mechanism involved in the inhibition of miR-29b-3p and pathogenesis and metastasis yet to be studied.

\section{Materials and methods}

\section{Cell culture and transfection}

Human breast cancer cell lines MDA-MB-231, MCF-7, MDA-MB-453 and human epithelial cell line MCF-10A were purchased from Procell (Wuhan, China). MDAMB-231, MCF-7, MDA-MB-453 were cultured in complete Dulbecco's modified Eagle's medium (DMEM, Gibco, USA) which supplemented with $10 \%$ fetal bovine serum (FBS, Gibco, USA), 1\% penicillin streptomycin (100 U/mL penicillin and $100 \mathrm{mg} / \mathrm{mL}$ streptomycin, $\mathrm{P} \& \mathrm{~S}$, Gibco, USA). MCF-10A was cultured in human epithelial cell complete medium (Procell, Wuhan, China) which containing DMEM/F12, horse serum, growth additive and $\mathrm{P} \& S$. All cell lines were cultured at $37^{\circ} \mathrm{C}$ in $5 \% \mathrm{CO}_{2}$ atmosphere. miR-29b-3p mimics and miR-29b-3p inhibitor, negative control, negative control inhibitor were purchased from GenePharma (Suzhou, China). To conduct cell transfection, cells were firstly cultured to about 50-60\% confluence. Then, Lipofectamine 2000 (Thermo Fisher Scientific, USA) was used and transfected according to the manufacturer's manual.

\section{Total RNA extraction and qRT-PCR (quantitative real-time polymerase chain reaction)}

Total RNAs were isolated from cells after $24 \mathrm{~h}$ transfection according to the manufacturer's protocol (AM1560, Invitrogen, USA), cDNA synthesis and miRNA expression were quantified using Mir- $\mathrm{X}^{\mathrm{TM}}$ miRNA qRT-PCR SYBR $^{\circledR}$ Kit (Takara, Japan) and Applied Biosystems 7500 (Thermo Fisher Scientific, USA) real time device under the standard thermal condition: $95^{\circ} \mathrm{C}$ for $30 \mathrm{~s}, 40$ cycles of $95^{\circ} \mathrm{C}$ for $10 \mathrm{~s}$ and $60^{\circ} \mathrm{C}$ for $30 \mathrm{~s}$, followed by $95^{\circ} \mathrm{C}$ for $10 \mathrm{~s}, 65^{\circ} \mathrm{C}$ for $60 \mathrm{~s}$ and $97^{\circ} \mathrm{C}$ for $1 \mathrm{~s}$. All results were normalized to U6. Using the $-2^{\triangle \triangle \mathrm{Ct}}$ method to identify the miRNA relative expression levels. Primer sequences: U6, forward primer: 5'-CTCGCTTCGGCAGCACA-3'; Reverse primer: 5'-AACGCTTCACGAATTTGCGT-3'; miR-29b-3p, forward primer: $5^{\prime}$-TAGCACCATTTGAAA TCAGTGTT- $3^{\prime}$, Reverse primer: mRQ $3^{\prime}$ Primer (CAS: 638313, Takara, Japan). Three independent experiments were performed for each reaction in triplicate. 


\section{Doxorubicin treatment}

Doxorubicin hydrochloride was purchased from Macklin (CAS: 25316-40-9, Shanghai, China). For Doxorubicin treatment, we prepared the Doxorubicin stocking solution at $1 \mathrm{mM}$ concentration in ultrapure water. Then, different concentration of Doxorubicin $(10 \mu \mathrm{M}, 1 \mu \mathrm{M}$, $0.1 \mu \mathrm{M}, 0.01 \mu \mathrm{M}, 0.001 \mu \mathrm{M}$ ) were dissolved in medium and treated for $24 \mathrm{~h}, 48 \mathrm{~h}$ and $72 \mathrm{~h}$ and CCK8 assay was performed, and IC50 of DOX was used for treatment to identify the miRNA relative expression.

\section{Cell proliferation assay}

Cell proliferation of MDA-MB-231 was detected using Cell Counting Kit-8 (CCK8) (Beyotime, Shanghai, China). Briefly, 8000 cells per well were seeded in 96-well plate with $100 \mu \mathrm{L}$ complete medium and cultured for $24 \mathrm{~h}$. After Doxorubicin or transfection treatment, $100 \mu \mathrm{L}$ medium containing $10 \mu \mathrm{L}$ CCK8 was added to the wells and incubated for $4 \mathrm{~h}$ later absorbance was measured using EnSpire Multimode Plate Reader (PerkinElmer, Waltham, Massachusetts, USA) at $450 \mathrm{~nm}$. Each group in triplicate.

\section{Colony formation assay}

Single cell suspension was obtained by trypsinization after transfection for $24 \mathrm{~h} .500$ cells were seeded in 6-well plate and cultured it for 2 weeks with complete medium in $5 \% \mathrm{CO}_{2}$ incubator. After colony formation, $4 \%$ paraformaldehyde (PFA) (Biosharp, Hefei, China) was used to fix the cells for 20 min and stained with $1 \%$ crystal violate (KeyGen BioTECH, Jiangsu, China) for $15 \mathrm{~min}$ at room temperature. The number of colony-forming units were calculated in three random wells.

\section{Migration assay}

To study the migration ability of MDA-MB-231 migration assay were conducted. Briefly, $5 \times 10^{5}$ MDA-MB-231 cells were seeded in 24-well and culture overnight. After 80-90\% confluence transient transfection was performed for $24 \mathrm{~h}$, later $1 \mu \mathrm{L}$ sterile tip was used to make a straight wound. Scratch photo was taken at $0 \mathrm{~h}, 6 \mathrm{~h}$ and $12 \mathrm{~h}$ by microscope (Olympus, Japan). Distance migration was measured by calculating the area of the wound using Image $J$ software and represented in the form of bar graph.

\section{Invasion assay}

To detect the invasive ability of MDA-MB-231 invasion assay was performed. After $24 \mathrm{~h}$ of transient transfection, $2.5 \times 10^{4}$ cells were harvested and seeded in the $8 \mu \mathrm{m}$ pore size upper Transwell chamber (Corning, USA) coated with Matrigel (CAS:356234, Corning, USA), which cultured in basic medium. The optimal coating concentration ratio was DMEM serum-free medium: Matrigel $=8: 1(\mathrm{~V} / \mathrm{V})$, and $2 \mathrm{~h}$ incubation at $37^{\circ} \mathrm{C} .900 \mu \mathrm{L}$ complete medium containing $5 \%$ FBS was added into the lower chamber as a chemoattractant. After $24 \mathrm{~h}$ incubation cells were fixed with $4 \%$ paraformaldehyde and stained with $1 \%$ crystal violate. Migratory cells in lower chamber were counted from each well and photographed at $20 \times$ (Olympus, Japan). The experiment was repeated three times.

\section{Immunofluorescence}

To study the cytoskeleton reorganization in breast cancer cell line MDA-MB 231 immunofluorescence technique was performed. $1 \times 10^{5}$ MDA-MB-231 cells were seeded on cover slips in 24 well plate. After $36 \mathrm{~h}$ of NC, miR-29b-3p mimics and miR-29b-3p inhibitor treated cells were fixed with $4 \%$ paraformaldehyde for $15 \mathrm{~min}$ at room temperature and then washed with PBS twice. Later, the cells were washed with a quenching solution (0.1\% glycine in PBS) twice, permeated with $0.1 \%$ Triton $\mathrm{X}-100$ for $10 \mathrm{~min}$ and blocked using a blocking solution (10\% FBS in PBS) for $1 \mathrm{~h}$ at room temperature. Later the cells were incubated for overnight with primary antibody against $\alpha$-Tubulin (anti $\alpha$-Tubulin (11H10), 1:100, 2125, Cell Signaling Technology, USA) followed by secondary antibody Alexa Fluor 594 Conjugated goat anti-rabbit IgG (1:50, ZF-0516, ZSGB-Bio, Beijing, China). Nuclei were stained with 4,6-diamidino-2 phenylindole DAPI and the cover slip was mounted using anti-fluorescent quencher (Beyotime, Shanghai, China). Immunofluorescence image were captured under LSM 710 Laser scanning confocal microscope (Carl Zeiss, German). Digital images were optimized for image resolution (final resolution $300 \mathrm{dpi}$ ), brightness, and contrast using Adobe Photoshop 7.0 (Adobe Systems, San Jose, CA, USA). No alteration was made in image such as adding or removal of image details.

\section{Hoechst staining}

To study the nuclear morphology of the MDA-MB-231 and MCF-7, Hoechst staining was performed. $1 \times 10^{5}$ MBA-MB-231 and MCF-7 cells were seeded on the cover slip in 24 well plate and transfected with NC, miR-29b-3p mimics and miR-29b-3p inhibitor for $36 \mathrm{~h}$. The cells were fixed for 20 min with $4 \%$ PFA then washed with PBS, incubated with $0.3 \%$ Triton-X 100 for 5 min. Later cells were washed and $100 \mu \mathrm{L}$ Hoechst 33258 (Beyotime, Shanghai, China) was added to cells and incubated for $30 \mathrm{~min}$ in dark. The cover slip was mounted using antifluorescent quencher (Beyotime, Shanghai, China). Photographed under the LSM 710 Laser scanning confocal microscope at $40 \times$ (Carl Zeiss, German). 


\section{TUNEL assay}

$1 \times 10^{5}$ MDA-MB-231 cells were seeded in 24-well plate and transfected with NC, miR-29b-3p mimics and miR29b-3p inhibitor for $36 \mathrm{~h}$. The cells were fixed with $4 \%$ paraformaldehyde for $30 \mathrm{~min}$ then wash with PBS. Later 0.3\% Triton-X 100 in PBS was added and incubated for $5 \mathrm{~min}$. One-step TUNEL apoptosis detection kit (Beyotime, Shanghai, China) was used to estimate the apoptosis efficiency according the manufacture's instruction. In brief, cells were incubated with $50 \mu \mathrm{L}$ TUNEL reaction buffer in a $37^{\circ} \mathrm{C}$ humidified chamber for $1 \mathrm{~h}$, nucleus was counterstained with DAPI for $1 \mathrm{~min}$ at room temperature in dark later mounted with anti-fade mounting medium. Stained apoptotic cells were visualized at $20 \times$ by LSM 710 Laser scanning confocal microscope (Carl Zeiss, German).

\section{Flow cytometry assay}

Annexin V/propidium iodide staining for apoptosis was conducted using Annexin V-FITC Detection kit (DojinDo, Japan) according to the manufacturer's instructions. Briefly, $1 \times 10^{6}$ MDA-MB-231 cells were seeded in 6 well plate after $24 \mathrm{~h}$ the cells were transfected with NC, miR-29b-3p mimics and miR-29b-3p inhibitor. The cells were trypsinized and single cells suspension was added with $5 \mu \mathrm{L}$ Annexin V-FITC followed by $5 \mu \mathrm{L}$ PI solution in dark and incubated for $15 \mathrm{~min}$. Soon $400 \mu \mathrm{L}$ Annexin V Binding Solution was added and analyzed by BD Accuri C6 Plus (BD) flow cytometer. Necrotic, early and late apoptosis cells were identified by BD Accuri C6 Plus software.

\section{Dual-luciferase reporter assay}

MDA-MB-231 cells were seeded into 24-well plates and after $24 \mathrm{~h}$ incubation the confluence reaches to $60-70 \%$. Psi-CHECK2/TRAF3-3'-UTR wt and Psi-CHECK2/ TRAF3-3'-UTR mutant reporter plasmids were constructed in advanced. According to the manufacturer's instruction, MDA-MB-231 cells were transiently cotransfected with miR-29b-3p mimics or miR-29b-3p inhibitor together with $0.1 \mu \mathrm{g}$ psi-CHECK2/TRAF33'-UTR wt or psi-CHECK2/TRAF3-3'-UTR mutant reporter plasmids using Lipofectamine 2000. After $48 \mathrm{~h}$, Dual-luciferase Reporter Assay System (CAS: E1910, Promega, USA) was used to detect firefly and Renilla luciferase activities and recorded using GloMax 96 Microplate Luminometer (Promega, USA).

\section{Western blot}

Breast cancer cell line MDA-MB-231 $\left(1 \times 10^{6}\right.$ cells/ each well in 6-well plates) was transfected with $\mathrm{NC}$, miR-29b-3p mimics and miR-29b-3p inhibitor for $48 \mathrm{~h}$. Cell lysates were collected using $1 \mathrm{~mL}$ RIPA (Solarbio,
Beijing, China) with $10 \mu \mathrm{L}$ PMSF. Protein concentration of cell lysates were measured with a BCA Protein Assay Kit (Sangon Biotech, Shanghai, China). Western blot was carried out by separating $40 \mu \mathrm{g}$ of protein by sodium dodecyl sulfate polyacrylamide gel electrophoresis (SDS-PAGE) and electro-transferred onto a polyvinylidene difluoride (PVDF) membrane (240 mA, $4 \mathrm{~h})$. The membranes were blocked with $5 \%$ non-fat milk and washed with TBST buffer and then incubated with primary antibody Cleaved PARP (Asp214) (D64E10), NF- $k B$ p65 (D14E12), phospho-NF-kB p65 (ser536), ІкB- $\alpha$ (44D4), phospho-ІкB- $\alpha$ (14D4), (Cell Signaling Technology, USA) at a dilution of 1:1000 in Primary Antibody Dilution Buffer (Beyotime. Shanghai, China) at $4{ }^{\circ} \mathrm{C}$ overnight. Next, membrane treated with primary antibody were washed 3 times with TBST and incubated with an anti-rabbit HRP-linked antibody at a 1:5000 dilutions for $1 \mathrm{~h}$ at room temperature, $\beta$-Actin was used as an internal reference. The signals were visualized by Immobilon Western Chemiluminescent HRP Substrate (Millipore Corporation, Billerica, MA, USA) with the Amersham Imager 600 imagers (GE healthcare Life science, Pittsburgh, USA).

\section{Statistical analysis}

Each experiment was repeated at least three times. All data were analyzed by using GraphPad Prism 6.01 and presented as mean \pm S.E.M. Differences between groups were considered significant at $\mathrm{p}<0.05$. We evaluated it by using the independent $\mathrm{t}$-test for the comparison of two samples and using a one-way ANOVA test for the comparison of more the two samples.

\section{Results}

miR-29b-3p was dysregulated in MDA-MB-231 and MCF-7

In order to identify the relative expression of miR-29b-3p between different breast cancer cell lines, we performed qRT-PCR in MDA-MB-231, MCF-7 and human epithelial cell line MCF-10A. The results show that miR-29b-3p was over-expressed significantly in TNBC cell line compared to ER negative cell line MCF-7 and MCF-10A cell line (Fig. 1B). We used Doxorubicin for different breast cancer cell lines and found that the cell viability was inhibited by it, which was concentration and time dependent (Fig. 1A). And after DOX treatment, the miR29b-3p was down-regulated in TNBC compare with before (Fig. 1C), which indicated that DOX regulated miR-29b-3p expression and miR-29b-3p may plays an important role in cell survival.

\section{MiR-29b-3p promotes TNBC cell proliferation in vitro}

To further investigate the biological function of miR29b-3p in TNBC. We performed CCK8 and colony 

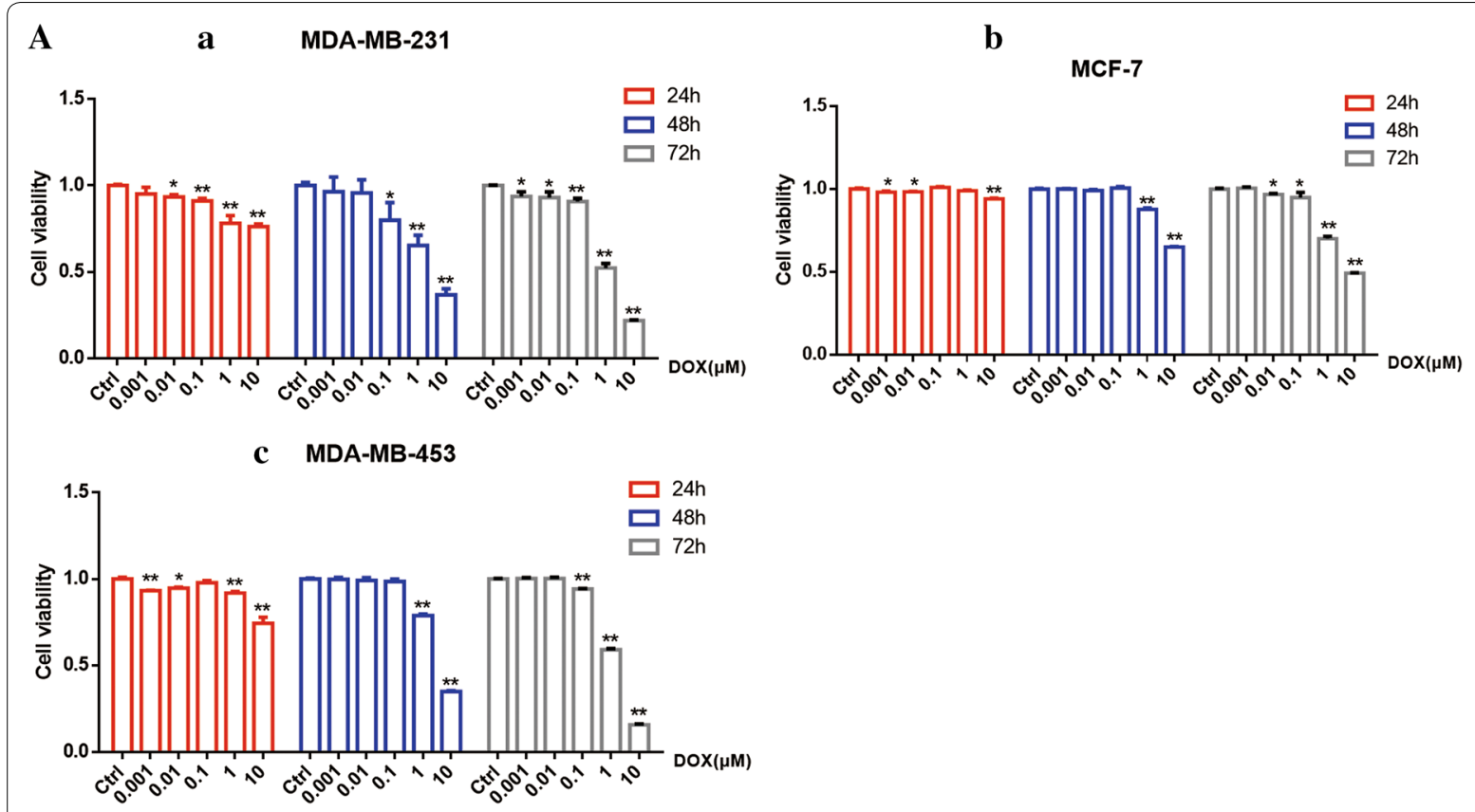

B Ralative expression of miR-29b-3p in MDA-MB-231 and MCF-7

C Relative expression of miR-29b-3p after DOX treatmented
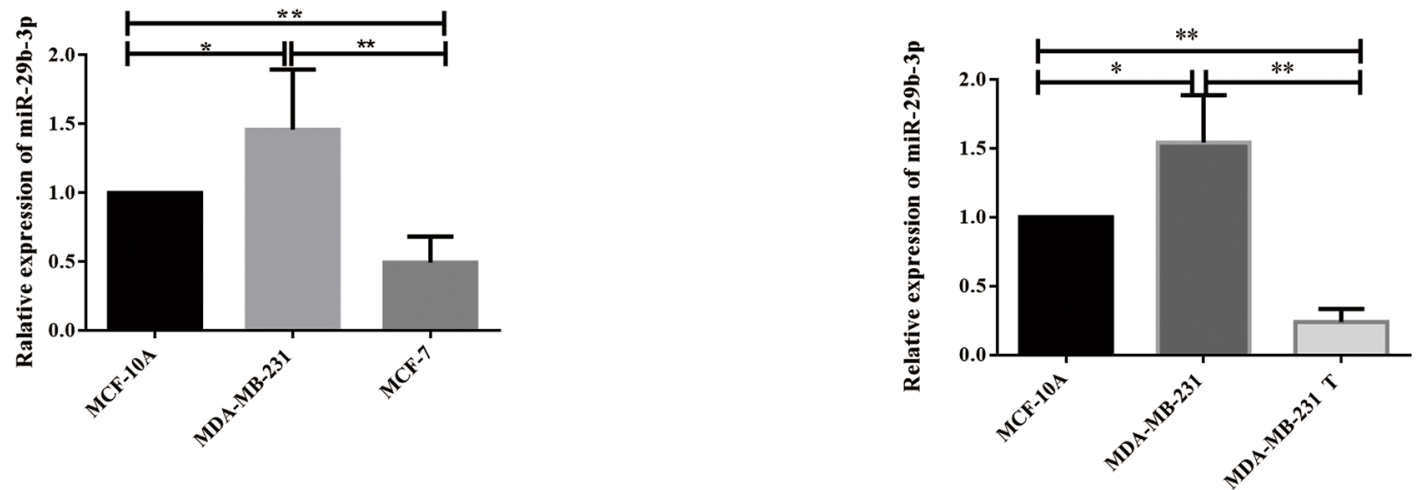

Fig. 1 The expression of miR-29b-3p in breast cancer cells. A Doxorubicin exhibits cytotoxic effect in breast cancer cells. (a) MDA-MB-231, (b) MCF-7 and (c) MDA-MB-453. The percentage inhibition of cell viability represented graphically. B Analysis of miR-29b-3p expression level in MDA-MB-231 and MCF-7 cells compared with MCF-10A cells. C Analysis of miR-29b-3p expression level in MDA-MB-231 after Doxorubicin treated. U6 was used as an internal control. $T$ treated. Data are presented as the mean \pm SD of three independent experiments. ${ }^{*} p<0.05,{ }^{* *} p<0.01$

formation assay to detect the cell viability. After transfection CCK8 assay was performed to show the influence of miR-29b-3p to TNBC. The results indicated that miR-29b-3p inhibitor inhibit the growth rate significantly compare with NC and miR-29b-3p mimics group. Besides, colony formation assay showed that miR-29b-3p inhibitor could effectively reduce the cell viability of MDA-MB-231, resulting in less cell colony formation than NC and miR-29b-3p mimics group (Fig. 2).
miR-29b-3p promotes MDA-MB-231 cell migration and invasion

MDA-MB-231 is highly aggressive cell line which exhibited a high migration and invasive capability. To assess the effects of miR-29b-3p on cell migration and invasion, migration assay and invasion assay were performed. The results suggested that overexpression of miR-29b-3p significantly promotes cell migration whereas suppressing the miR-29b-3p expression inhibits cell migration process (Fig. 3a). Comparable results were obtained from the invasion assay. The number of invasive cells significantly decreases in miR-29b-3p inhibitor group compared with 

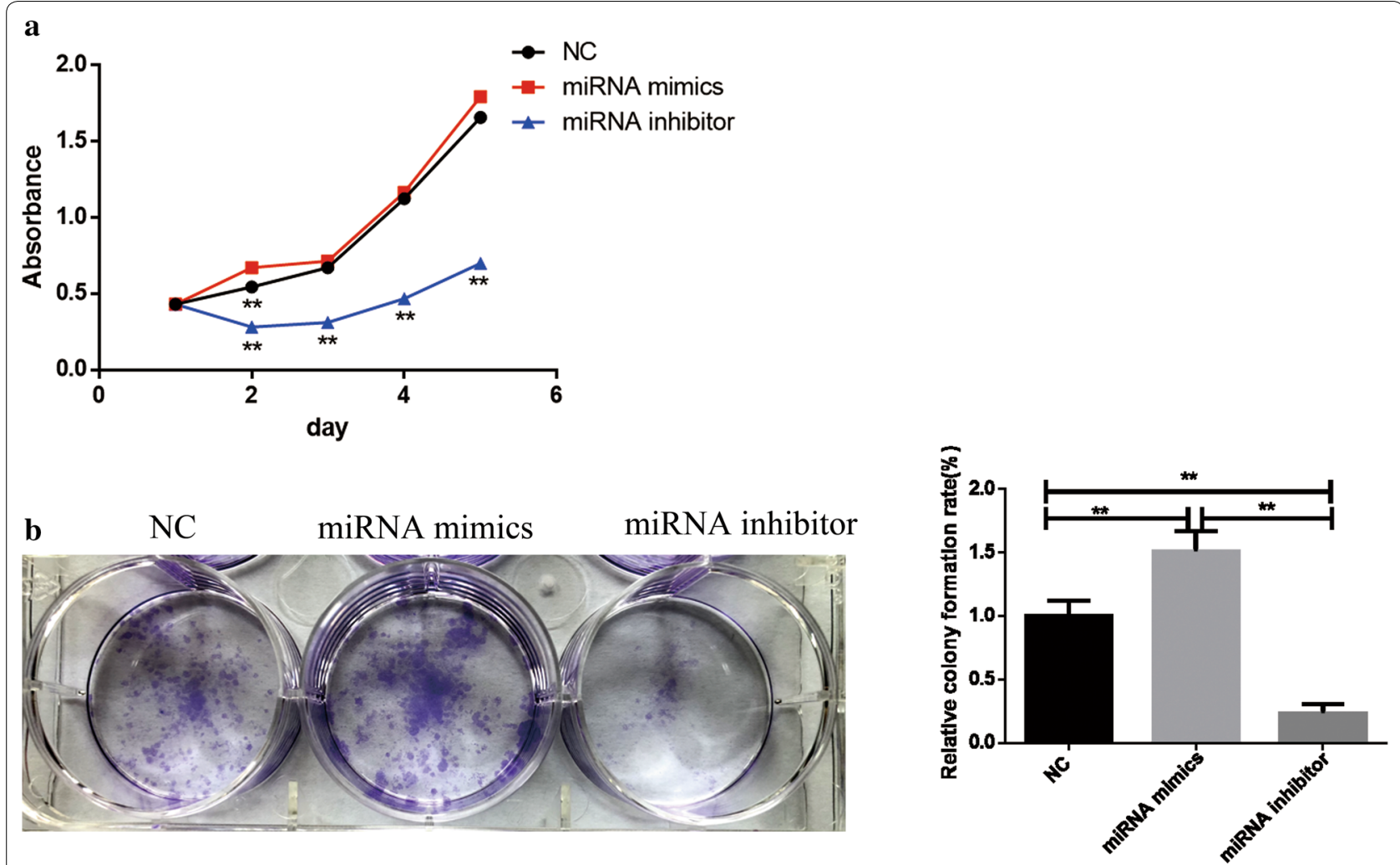

Fig. 2 Cell proliferation and colony forming ability of MDA-MB-231 cells. a CCK-8 assay was applied to determine the activity of cells after transfection. $\mathbf{b}$ miR-29b-3p inhibitor inhibited cell colony formation in MDA-MB-231 cells. Data are presented as the mean \pm SD of three independent experiments. ${ }^{*} p<0.05,{ }^{* *} p<0.01$

NC and miR-29b-3p mimics group (Fig. 3b). The quantified results showed that miR-29b-3p inhibitor inhibits cell migration and invasion in MDA-MB-231. To study the cell integrity in the presence of miR-29b-3p inhibitor treatment we stained cells with the anti- $\alpha$-tubulin antibody. The results revealed that miR-29b-3p inhibitor treatment damages the cytoskeleton and destroy cell integrity and probably affecting cell mobility (Fig. 3c).

\section{Inhibition of miR-29b-3p induces apoptosis in TNBC breast} cancer cell line

To evaluate the apoptosis induced by miR-29b-3p inhibitor in MDA-MB-231, we performed TUNEL assay, Annexin V-FITC/PI staining, nuclear staining and Western Blot. MDA-MB-231 cells were transiently transfected with NC, miR-29b-3p mimics and miR-29b-3p inhibitor and stained with probe labelled with Cy3. As shown in Fig. 4A, fluorescence of Cy3 labeled probe was more in miR-29b-3p inhibitor group than NC and miR-29b-3p mimics group. In addition, Annexin V-FITC/PI staining was performed to determine direct effects of miR-29b-3p mimic and miR29b-3p inhibitor on MDA-MB-231 breast cancer cells
(Fig. 4B). The results showed that percentage of cell apoptosis in miR-29b-3p inhibitor group increased compare with $\mathrm{NC}$ and miR-29b-3p mimics group. To examine the nuclear morphology of apoptotic cells nuclear staining was conducted using Hoechst staining. The results showed that miR-29b-3p inhibitor increases the nuclear fragmentation and cell blebbing in MDAMB-231 and MCF-7 cells (Fig. 4C). Further we isolated the total proteins and performed Western blot for poly ADP-ribose polymerase (PARP). Compared with the NC group, the miR-29b-3p inhibitor group exhibited an increased protein level of cleaved PARP while expression level in miR-29b-3p mimics group was less (Fig. 4D). Taken together, inhibition of miR-29b-3p will induce apoptosis in MDA-MB-231.

\section{miR-29b-3p targets to $3^{\prime}$-UTR of TRAF3 and regulates NF-KB signaling pathway in breast cancer}

To identify the putative mechanisms of miR-29b-3p mediated regulation of MDA-MB-231, bioinformatics predictions were performed by using Targetscan, PicTar, miRDB. We found that miR-29b-3p targets to TRAF3 (Fig. 5a). Subsequently, Dual-luciferase reporter assays 


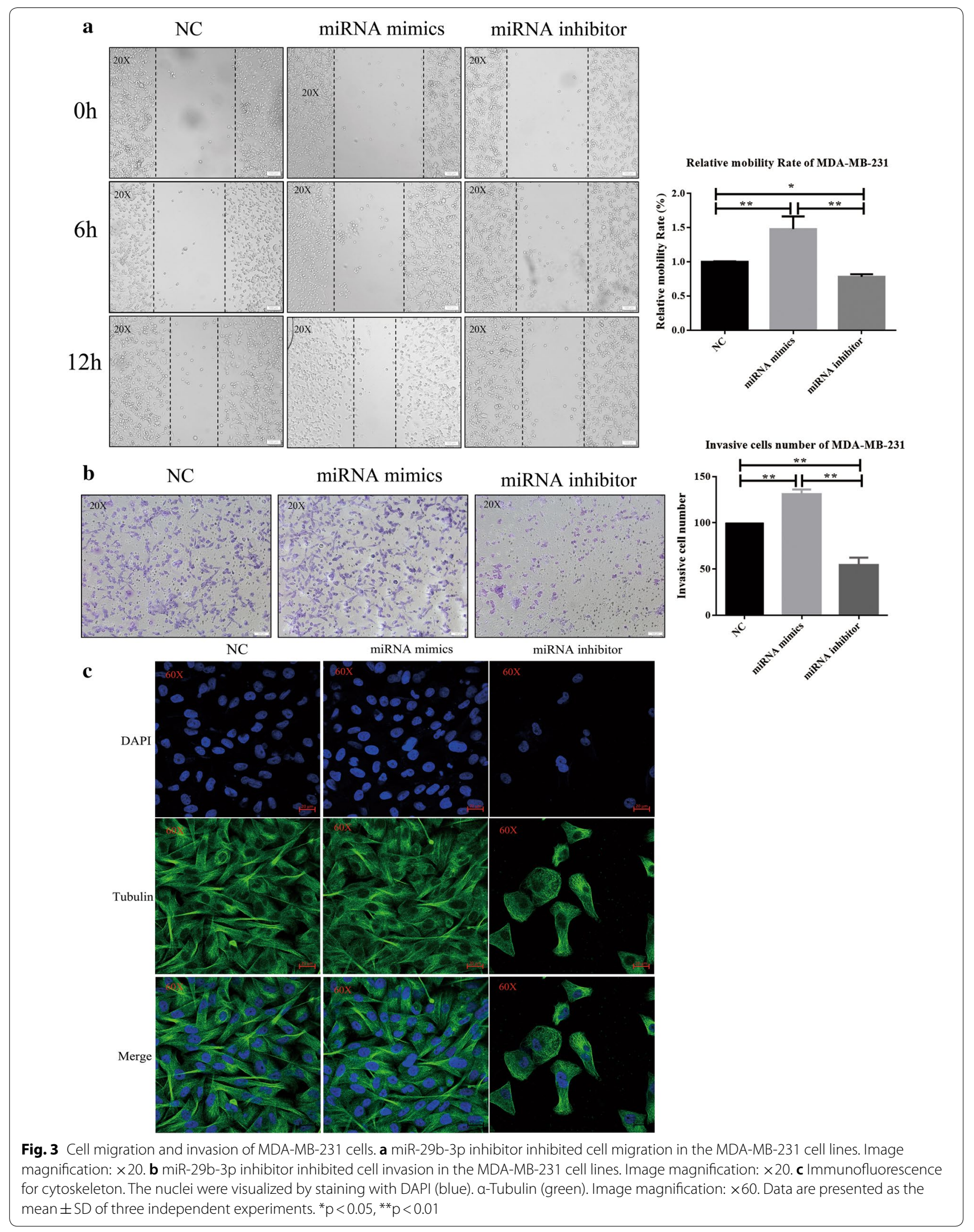




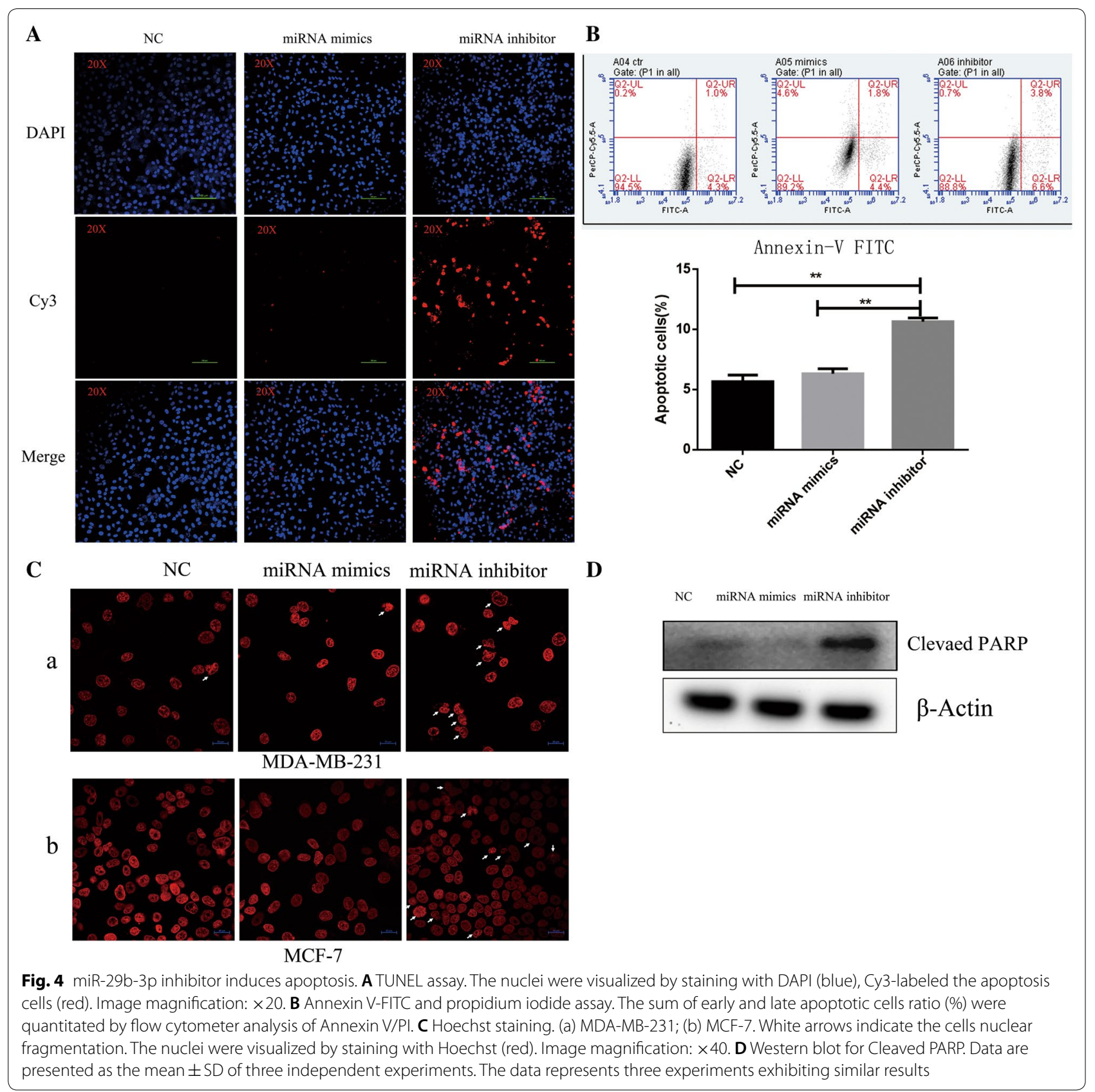

were performed to determine the relative luciferase activity in NC, miR-29b-3p mimics and miR-29b-3p inhibitor with TRAF3 3'-UTR wt or TRAF3 3'-UTR mt reporter plasmids. As shown in Fig. 5b, c, significant decrease in relative luciferase activity was noted when psi-CHECK2/ TRAF3 3'-UTR wt was co-transfected with the miR29b-3p mimics, while the relative luciferase activity in miR-29b-3p mimic group with psi-CHECK2/TRAF3 $3^{\prime}$-UTR mt reporter plasmid had no significant difference with the NC group with psi-CHECK2/TRAF3 3 '-UTR mt. Moreover, the relative luciferase activity in miR29b-3p inhibitor group with TRAF3 3'-UTR wt reporter plasmid was markedly higher than the NC group with TRAF3 3'-UTR wt, while the relative luciferase activity in miR-29b-3p inhibitor group with TRAF3 $3^{\prime}$-UTR wt reporter plasmid had no significant difference with the NC group with TRAF3 $3^{\prime}$-UTR wt. Therefore, TRAF3 is one target gene of miR-29b-3p. To further understand the mechanism involved in miR-29b-3p regulating NF- $\mathrm{kB}$ pathway we performed Western blot experiment. As 


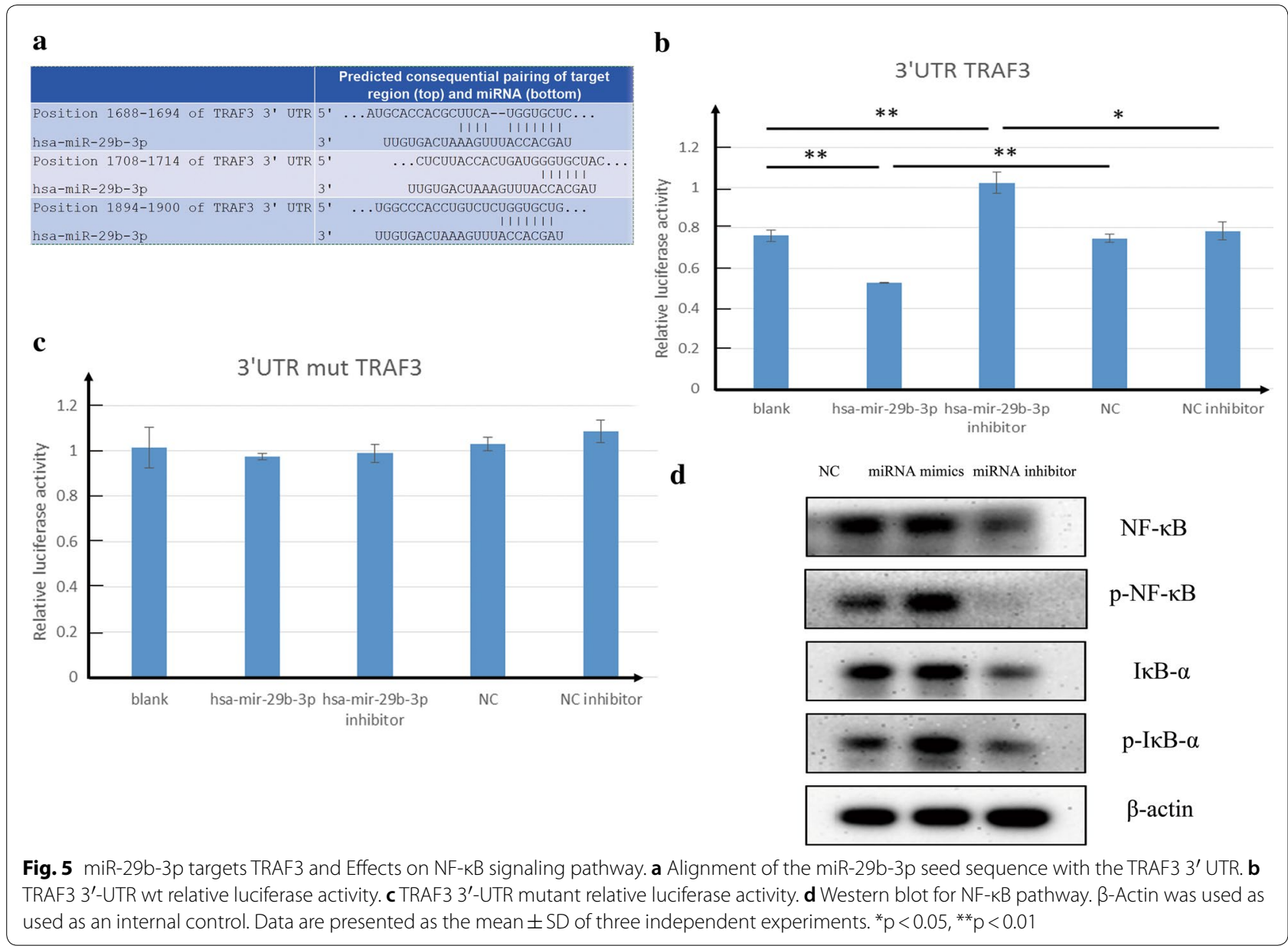

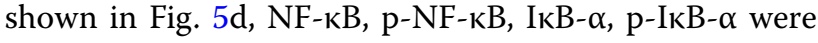
decreased after miR-29b-3p inhibitor treatment whereas the miR-29b-3p mimics group was increased significantly compared with NC group. It indicated that miR-29b-3p induced activation of NF- $\mathrm{kB}$ signaling pathway and apoptosis resistance.

\section{Discussion}

Breast cancer is a common malignant cancer among women worldwide. Triple negative breast cancer is more aggressive and has a higher mortality rate due to lack of effective treatments. Cumulative studies have demonstrated that miRNAs are molecular regulators in different diseases [33-36]. Our results found that miR-29b-3p was overexpressed in MDA-MB-231 while low-expressed in MCF-7 in compassion to MCF-10A. To explore the reasons behind this phenomenon, we used Doxorubicin (DOX), a well-known antitumor drug as positive control to induce apoptosis [37] in MDA-MB-231 cells and performed qRT-PCR. After DOX treatment, the miR29b-3p expression was decreased significantly compare with non-treated MDA-MB-231 cells. The results indicated that miR-29b-3p may be involved in the progression and survival of breast cancer. In order to investigate the biological relevance of miR-29b-3p in breast cancer. miR-29b-3p inhibitor was transfected into MDA-MB-231 cells. Our results suggested that miR-29b-3p inhibitor inhibits cell proliferation, migration and invasion significantly in vitro. Cancer cell migration and invasion are responsible for the most number of cancer incidents [38, 39]. In this process, cancer cells acquired migratory and invasive capabilities and then migrator to distant site, this spreads the cancer cells throughout the body to seed secondary tumors at distant sites. It's a complex process that involved a dramatic reorganization of cell cytoskeleton [40, 41]. Cell cytoskeleton refers to the protein fiber network system in eukaryotic cells which composed of microtubule (MT), microfilament (MF) and intermediate filament (IF). It has been reported that alterations in the cell cytoskeleton was associated with metastasis, invasion and apoptosis [42-45]. Herein, we performed immunofluorescence to check the formation of microtubule, our results showed that miR-29b-3p inhibitor has 
an influence on the formation of the microtubule which destroys cell integrity and probably affecting cell mobility, Consequently, miR-29b-3p is a tumor promoter in TNBC.

Next, we predicated and validated the possible target mRNAs of miR-29b-3p. Previous studies have shown that miR-29b-3p targets many genes and exerts different biological functions, such as MCMBP, PI15, COL3A1 (TargetScan v7.2; http://www.targetscan.org). Therefore, based on the TargetScan predictions, it showed that TRAF3 is targeted by miR-29b-3p. Dual-luciferase reporter assay showed that significant decrease in relative luciferase activity was noted when psi-CHECK2/ TRAF3-3'-UTR was co-transfected with the miR-29b-3p mimics compare with $\mathrm{NC}$ group, it indicated that miR29b-3p mimics bind to the 3'UTR of the TRAF3, thereby inhibiting the luciferase activity. However, the relative luciferase activity was increased when psi-CHECK2/ TRAF3 3'-UTR was co-transfected with the miR29b-3p inhibitor compare with NC group. It indicated that miR-29b-3p inhibitor inhibits the endogenous miR-29b-3p binding to the TRAF3 $3^{\prime}$-UTR, resulting in stronger relative luciferase activity than the $\mathrm{NC}$ group. But in miR-29b-3p mimics group, the relative luciferase activity was decreased compare with NC group. Moreover, no significant difference among groups when psiCHECK2/TRAF3 3'-UTR mutant co-transfected with NC, miR-29b-3p mimics and miR-29b-3p inhibitor. The results showed that miR-29b-3p binds to TRAF3 and miR-29b-3p inhibitor inhibited miR-29b-3p. Therefore, TRAF3 is one target gene of miR-29b-3p. TRAF3, one of the six members of this family of proteins in humans and mice, has been reported to interact with other members of the TNFR family $[46,47]$ and mediate the signal transduction, such as CD40 and LMP-1. These two members are important for the activation of the immune response [48]. Different from many other TRAF family proteins that enhance NF- $\mathrm{KB}$ activation, TRAF3 has been reported to inhibit the activation of NF- $\mathrm{kB}$ induced by TNFR family [49] and was identified as a negative regulator of NF- $\mathrm{KB}$ inducing kinase (NIK), promoting its degradation $[50,51]$. Activation of NF- $\mathrm{kB}$ signaling pathway is mediated by TNFR and it involved many biologic processes, such as cell proliferation, apoptosis and immune responses [52, 53]. During apoptosis, NF- $\mathrm{kB}$ signaling pathway is inhibited and expression of Bcl-2 is downregulated mediating the mitochondrial apoptotic pathway which release cytochrome $C$ followed by caspase 9 and caspase 3 activation ultimately leading to PARP cleavage [54]. In accordance with the above studies, our results showed that miR-29b-3p inhibitor induces apoptosis and DNA fragmentation in MDA-MB-231, it suppresses NF- $\mathrm{B}$ s signaling pathway and induces PARP cleavage.

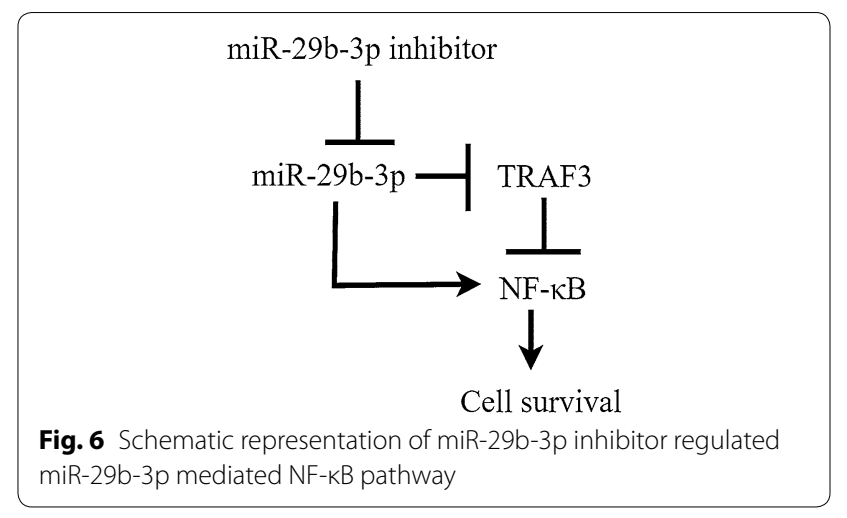

Besides, over-expression of miR-29b-3p in TNBC resulting in inhibition of TRAF3 and activation of NF-KB signaling pathway. However, miR-29b-3p inhibitor can inhibit miR-29b-3p binding to TRAF3 (Fig. 6).

Taken together, we identified miR-29b-3p as an important regulator in TNBC which enhances cell proliferation, migration and invasion. Moreover, inhibition of miR$29 \mathrm{~b}-3 \mathrm{p}$ induces cell apoptosis by suppressing NF- $\mathrm{kB}$ signaling pathway in MDA-MB-231. Altogether our results showed that miR-29b-3p has a key role in MDA-MB-231.

\section{Conclusion}

Our findings indicated that miR-29b-3p was highly expressed in MDA-MB-231 cells. Inhibition of miR29b-3p could suppress the cancer cells proliferation and induced the apoptosis. In addition, it influenced the formation of microtubule and suppressed the process of cell migration and invasion. Moreover, miR-29b-3p targets to the $3^{\prime}$-UTR of TRAF3 and activates the NF- $\mathrm{kB}$ signaling pathway. Therefore, miR-29b-3p could be an underlying biomarker and it can be developed as novel therapeutic target against the TNBC.

\section{Abbreviations \\ miRNAs: microRNAs; NF-kB: nuclear factor kappa B; 3'-UTR: 3'-untranslated region; TNBC: triple negative breast cancer; qPCR: quantitative PCR; DMEM: Dulbecco's modified Eagle medium; FBS: fetal bovine serum; PARP: poly ADP-ribose polymerase; HRP: horse radish peroxidase; TRAF3: TNF receptor- associated factor 3; SD: standard deviation.}

\section{Acknowledgements}

We thank Prof. Kun Wei for supporting. We thank members of our laboratory for many discussions and helpful comments on the manuscript.

\section{Authors' contributions}

BZ planned experiments; All the authors performed experiments; BZ analyzed data and wrote the draft of paper; DS and CF revised the paper. All the authors accepted the final version of the paper. All authors read and approved the final manuscript. 


\section{Funding}

This study was supported by the International Cooperation Projects of Guangdong Provincial Science and Technology (Grant no. 2015A050502013), Multistage construction of high-intensity microsphere scaffolds and development of bone repair materials (Grant no. 2015A020214005)

\section{Availability of data and materials}

Please contact the corresponding author for data on reasonable request.

\section{Ethics approval and consent to participate}

Not applicable.

\section{Consent for publication}

All the authors agreed to publish the paper.

\section{Competing interests}

The authors declare that they have no competing interests.

Received: 11 May 2019 Accepted: 19 July 2019

Published online: 26 July 2019

\section{References}

1. Banerji S, et al. Sequence analysis of mutations and translocations across breast cancer subtypes. Nature. 2012;486(7403):405-9.

2. Siegel RL, Miller KD, Jemal A. Cancer statistics, 2017. CA Cancer J Clin. 2017;67:7-30. https://doi.org/10.3322/caac.21387.

3. Fertig EJ, et al. Analysis of gene expression of secreted factors associated with breast cancer metastases in breast cancer subtypes. Sci Rep. 2015;5:12133.

4. Fan L, et al. Breast cancer in China. Lancet Oncol. 2014;15(7):e279-89.

5. Survcan WHO. Cancer survival in Qidong C, 1992-2000. http://survc an.iarc.fr/survival/chap7.pdf. Accessed 15 Mar 2019.

6. Survcan WHO. Cancer survival in Shanghai C, 1992-1995. http://survc an.iarc.fr/survival/chap7.pdf. Accessed 15 Mar 2019.

7. Marotti JD, et al. Triple-negative breast cancer: next-generation sequencing for target identification. Am J Pathol. 2017;187(10):2133-8.

8. Lehmann $\mathrm{BD}$, et al. Identification of human triple-negative breast cancer subtypes and preclinical models for selection of targeted therapies. J Clin Invest. 2011;121(7):2750-67.

9. Tormo $\mathrm{E}$, et al. The miRNA-449 family mediates doxorubicin resistance in triple-negative breast cancer by regulating cell cycle factors. Sci Rep. 2019;9(1):5316.

10. Khordadmehr M, et al. Key microRNAs in the biology of breast cancer; emerging evidence in the last decade. J Cell Physiol. 2019;234(6):8316-26.

11. Seeley JJ, et al. Induction of innate immune memory via microRNA targeting of chromatin remodelling factors. Nature. 2018;559(7712):114-9.

12. Li S, et al. MicroRNA-21: a ubiquitously expressed pro-survival factor in cancer and other diseases. Mol Cell Biochem. 2012;360(1):147-58.

13. Acunzo M, et al. MicroRNA and cancer-a brief overview. Adv Biol Regul. 2015;57:1-9.

14. Lee $Y$, et al. MicroRNA genes are transcribed by RNA polymerase II. EMBO J. 2004;23(20):4051-60.

15. Cheng AM, et al. Antisense inhibition of human miRNAs and indications for an involvement of miRNA in cell growth and apoptosis. Nucleic Acids Res. 2005:33(4):1290-7.

16. Goldar S, et al. Molecular mechanisms of apoptosis and roles in cancer development and treatment. Asian Pac J Cancer Prev. 2015;16(6):2129-44

17. Calin GA, et al. Frequent deletions and down-regulation of micro-RNA genes miR15 and miR16 at 13q14 in chronic lymphocytic leukemia. Proc Natl Acad Sci USA. 2002;99(24):15524-9.

18. Zhang BG, et al. microRNA-21 promotes tumor proliferation and invasion in gastric cancer by targeting PTEN. Oncol Rep. 2012;27(4):1019-26.

19. Sun EH, Zhou Q, Liu K-S, Wei W, Wang C-M, Liu X-F, Lu C, Ma D-Y. Screening miRNAs related to different subtypes of breast cancer with miRNAs microarray. Eur Rev Med Pharmacol Sci. 2014;18(19):2783-8.

20. Sun J, et al. Identification and profiling of conserved and novel microRNAs from Chinese Qinchuan bovine longissimus thoracis. BMC Genomics. 2013;14:42
21. Hu J, et al. Identification of microRNA-93 as a functional dysregulated miRNA in triple-negative breast cancer. Tumour Biol. 2015;36(1):251-8.

22. Adhami $M$, et al. Candidate miRNAs in human breast cancer biomarkers: a systematic review. Breast Cancer. 2018;25(2):198-205.

23. Zhang XM, et al. MicroRNA-29b promotes the adipogenic differentiation of human adipose tissue-derived stromal cells. Obesity. 2016:24(5):1097-105

24. Maegdefessel $L$, et al. Inhibition of microRNA-29b reduces murine abdominal aortic aneurysm development. J Clin Invest. 2012;122(2):497-506

25. Yang CN, et al. MicroRNA-29b regulates migration in oral squamous cell carcinoma and its clinical significance. Oral Oncol. 2015;51(2):170-7.

26. Liu B, et al. MiR-29b/Sp1/FUT4 axis modulates the malignancy of leukemia stem cells by regulating fucosylation via Wnt/beta-catenin pathway in acute myeloid leukemia. J Exp Clin Cancer Res. 2019;38(1):200.

27. Langsch S, et al. miR-29b mediates NF-kappaB signaling in KRAS-induced non-small cell lung cancers. Cancer Res. 2016;76(14):4160-9.

28. Encarnacion-Medina J, et al. MicroRNA expression changes in women with breast cancer stratified by DNA repair capacity levels. J Oncol. 2019;2019:7820275.

29. Yan B, et al. The role of miR-29b in cancer: regulation, function, and signaling. Onco Targets Ther. 2015;8:539-48.

30. Jiang $\mathrm{H}$, et al. Diverse roles of miR-29 in cancer (review). Oncol Rep. 2014;31(4):1509-16.

31. Han YC, et al. microRNA-29a induces aberrant self-renewal capacity in hematopoietic progenitors, biased myeloid development, and acute myeloid leukemia. J Exp Med. 2010;207(3):475-89.

32. Nguyen T, et al. Downregulation of microRNA-29c is associated with hypermethylation of tumor-related genes and disease outcome in cutaneous melanoma. Epigenetics. 2014;6(3):388-94.

33. Esteller M. Non-coding RNAs in human disease. Nat Rev Genet. 2011;12(12):861-74

34. Rupaimoole R, Slack FJ. MicroRNA therapeutics: towards a new era for the management of cancer and other diseases. Nat Rev Drug Discov. 2017; 16(3):203-22.

35. Rupaimoole R, et al. miRNA deregulation in cancer cells and the tumor microenvironment. Cancer Discov. 2016;6(3):235-46.

36. Majumder S, Jacob ST. Emerging role of microRNAs in drug-resistant breast cancer. Gene Expr. 2018;15(3):141-51.

37. Jose $P$, et al. Doxorubicin-loaded nanoparticles: new advances in breast cancer therapy. Anticancer Agents Med Chem. 2012;12(9):1058-70.

38. Gueron G, Siervi AD, Vazquez E. Key questions in metastasis: new insights in molecular pathways and therapeutic implications. Curr Pharm Biotechnol. 2011;12(11):1867-80.

39. Schroeder A, et al. Treating metastatic cancer with nanotechnology. Nat Rev Cancer. 2011;12(1):39-50.

40. Sens P, Plastino J. Membrane tension and cytoskeleton organization in cell motility. J Phys Condens Matter. 2015;27(27):273103.

41. Raudenska $M$, et al. Cisplatin enhances cell stiffness and decreases invasiveness rate in prostate cancer cells by actin accumulation. Sci Rep. 2019;9(1):1660.

42. Yilmaz M, Christofori G. EMT, the cytoskeleton, and cancer cell invasion. Cancer Metastasis Rev. 2009;28(1-2):15-33.

43. Fife CM, McCarroll JA, Kavallaris M. Movers and shakers: cell cytoskeleton in cancer metastasis. Br J Pharmacol. 2014;171(24):5507-23.

44. Yamaguchi $\mathrm{H}$, Condeelis J. Regulation of the actin cytoskeleton in cancer cell migration and invasion. Biochim Biophys Acta. 2007;1773(5):642-52.

45. Yang $\mathrm{R}-\mathrm{H}$, et al. EGFL7-overexpressing epidermal stem cells promotes fibroblast proliferation and migration via mediating cell adhesion and strengthening cytoskeleton. Mol Cell Biochem. 2016;423(1):1-8.

46. He JQ, et al. TRAF3 and its biological function, in TNF receptor associated factors (TRAFs). In: Wu H, editor. New York: Springer New York. 2007. p. 48-59.

47. Zapata JM, Lefebvre S, Reed JC. Targeting TRAFs for therapeutic intervention, in TNF receptor associated factors (TRAFs). In: Wu H, editor. New York: Springer New York; 2007. p. 188-201.

48. Zou M, Wang F, Jiang A, Xia A, Kong S, Gong C, Zhu M, Zhou X, Zhu J, Zhu W, Cheng W. MicroRNA-3178 ameliorates inflammation and gastric carcinogenesis promoted by Helicobacter pylori new toxin, Tip-a, by targeting TRAF3. Helicobacter. 2017;22:e12348. https://doi.org/10.1111/ hel.12348 
49. Hauer J, et al. TNF receptor (TNFR)-associated factor (TRAF) 3 serves as an inhibitor of TRAF2/5-mediated activation of the noncanonical NF-kappaB pathway by TRAF-binding TNFRs. Proc Natl Acad Sci USA. 2005;102(8):2874-9.

50. Liao G, et al. Regulation of the NF-kappaB-inducing kinase by tumor necrosis factor receptor-associated factor 3 -induced degradation. J Biol Chem. 2004:279(25):26243-50.

51. Zapata JM, et al. Lymphocyte-specific TRAF3 transgenic mice have enhanced humoral responses and develop plasmacytosis, autoimmunity, inflammation, and cancer. Blood. 2009;113(19):4595-603.

52. Karin M, Lin A. NF-KB at the crossroads of life and death. Nat Immunol. 2002;3:221.
53. Yang J, et al. CBP knockdown inhibits angiotensin Il-induced vascular smooth muscle cells proliferation through downregulating NF-kB transcriptional activity. Mol Cell Biochem. 2010;340(1):55-62.

54. Marsden VS, et al. Apoptosis initiated by BCl-2-regulated caspase activation independently of the cytochrome c/Apaf-1/caspase-9 apoptosome. Nature. 2002;419(6907):634-7.

\section{Publisher's Note}

Springer Nature remains neutral with regard to jurisdictional claims in published maps and institutional affiliations.
Ready to submit your research? Choose BMC and benefit from:

- fast, convenient online submission

- thorough peer review by experienced researchers in your field

- rapid publication on acceptance

- support for research data, including large and complex data types

- gold Open Access which fosters wider collaboration and increased citations

- maximum visibility for your research: over $100 \mathrm{M}$ website views per year

At BMC, research is always in progress.

Learn more biomedcentral.com/submissions 